\title{
Ostéosarcome primitif du rein avec évolution métastatique au foie. Étude de cas et revue de la littérature
}

\author{
Olfa Gharbi, MD; ${ }^{*}$ Amel Trabelsi, MD; ${ }^{\dagger}$ Makram Hochlef, MD; ${ }^{*}$ Soulef Kriaa, MD; ${ }^{+}$Sami Limam, MD; ${ }^{*}$ \\ Leila Ben Fatma, MD; ${ }^{*}$ Amel Landolsi, MD; ${ }^{*}$ Selma Gahbiche, MD; ${ }^{*}$ Moncef Mokni, MD; ; T.A. Mosbeh, MD; ${ }^{\xi}$ \\ Slim Ben Ahmed, MD*
}

\section{Résumé}

L'ostéosarcome primitif du rein est une pathologie très rare dont I'histogénèse n'est pas claire. Les récidives locales et les métastases sont fréquentes et le pronostic est très mauvais. Nous décrivons ici le cas d'une patiente de 59 ans qui présentait des douleurs lombaires gauches et un amaigrissement. Une échographie abdominale et une tomodensitométrie abdominale ont révélé une masse rétropéritonéale de $13 \times 8 \times 9 \mathrm{~cm}$ avec macrocalcifications. Le bilan d'extension n'a pas révélé d'autres lésions. On a procédé à une néphrectomie élargie gauche; I'examen histologique a révélé une prolifération tumorale constituée de cellules fusiformes de taille variable, ces cellules manifestement atypiques produisant directement du tissu ostéoïde évoquant un ostéosarcome rénal. Dix mois plus tard, la patiente présentait des lésions métastatiques au niveau hépatique sans autre lésion secondaire ni récidive locale. La patiente est actuellement en cours de traitement par chimiothérapie par doxorubicine et cisplatine avec un recul de 17 mois. Les caractéristiques anatomocliniques et les modalités thérapeutiques de cette pathologie rare sont discutées ci-dessous.

Can Urol Assoc J 2009;3(2):163-6

\section{Abstract}

Primary osteosarcoma of the kidney is very rare. Its exact histogenesis remains unclear. It has a tendency to recur locally and metastasize, and the prognosis is very poor. We present a case of a 59-year-old woman with left flank pain and weight loss. Abdominal ultrasonography and bone scan revealed a large solid retroperitoneal mass with calcifications. The patient underwent radical nephrectomy; microscopic examination showed atypical cells with the characteristic pattern of classic osteosarcoma with immature neoplastic osteoid. Ten months later, the patient developed metastatic lesions in the liver, without local recurrence or other sites of metastases. The patient is currently receiving chemotherapy with doxorubicin and cisplatin, with a follow-up in 17 months. The most important clinical findings, the pathogenesis and the treatment modalities of this rare neoplasm are discussed.

\section{Introduction}

L'ostéosarcome primitif du rein est une pathologie très rare; une vingtaine de cas seulement ont été signalés dans la littérature. Le pronostic est très mauvais et I'histogénèse n'est pas claire. Les récidives locales et les métastases sont fréquentes et la durée médiane de la survie est de 18 mois. Le diagnostic est le plus souvent posé à un stade avancé. Le traitement palliatif de cette pathologie repose sur la chirurgie, la chimiothérapie et la radiothérapie ${ }^{1,2}$.

Nous décrivons ici un cas d'ostéosarcome primitif du rein avec évolution non favorable survenu 1 an et demi après I'ablation chirurgicale de métastases hépatiques.

\section{Observations}

Une patiente de 59 ans présentant des antécédents de diabète non insulinodépendant mais pas d'antécédents familiaux de néoplasie a consulté au mois de juillet 2006 en raison de douleurs lombaires gauches avec un amaigrissement non chiffré, sans signe urinaire bas. L'examen a révélé une sensibilité à la palpation de la fosse lombaire gauche sans masse évidente. Le bilan biologique était normal et l'échographie abdominale montre une image évoquant une masse tissulaire rénale gauche polylobée, d'échostructure hétérogène, vascularisée en Doppler, avec calcifications et mesurant $9 \times 7 \mathrm{~cm}$. Cet examen a été suivi d'une tomodensitométrie (TDM) abdominale qui a révélé au niveau du pôle supérieur du rein gauche une masse rétropéritonéale de $13 \times 8 \times 9 \mathrm{~cm}$, bien délimitée, de densité hétérogène et avec macrocalcifications. Cette masse se rehaussait de façon progressive après injection d'un produit de contraste, avec des zones de nécrose centrale. La masse refoule le pancréas et l'artère splénique vers l'avant sans les envahir (Fig. 1). Le bilan d'extension n'a pas révélé d'autres lésions osseuses ou viscérales. La patiente a été opérée en octobre 2006 en raison d'une tumeur rénale gauche; on a procédé à une néphrectomie élargie gauche. L'examen anatomopathologique a révélé une formation tumorale calcifiée de $13 \times 11 \times 8 \mathrm{~cm}$ avec de multiples foyers nécroticohémorragiques; l'examen histologique a montré qu'il s'agissait d'une prolifération tumorale composée de cellules fusiformes de taille variable (Fig. 2) infiltrant la capsule et 
Gharbi et al.

la graisse rénales; ces cellules fusiformes sont associées à des cellules géantes de type ostéoclaste et produisent du tissu ostéoïde (Fig. 3). Les cellules tumorales sont manifestement atypiques (Fig. 4);

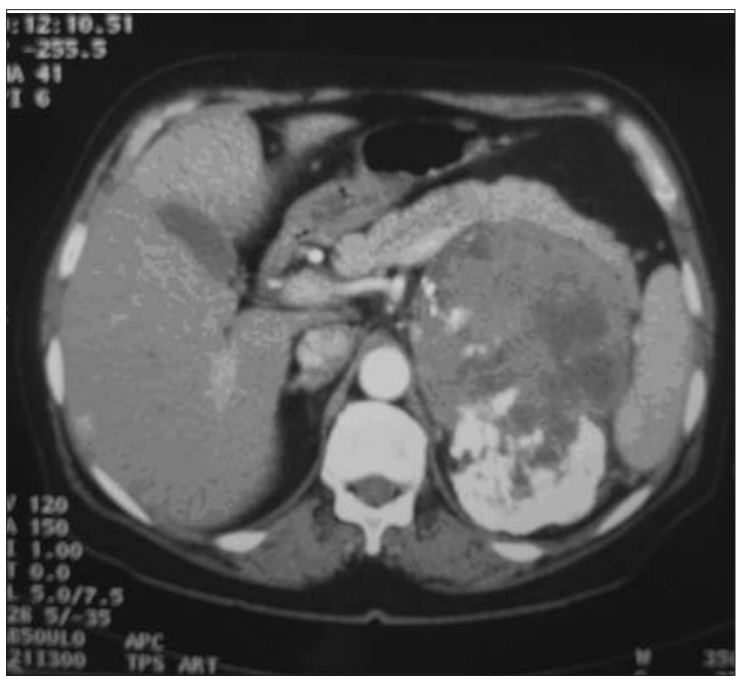

Fig. 1. Tomodensitométrie abdominale : masse au niveau du pôle supérieur du rein gauche, de densité hétérogène, avec macrocalcifications et nécrose centrale.

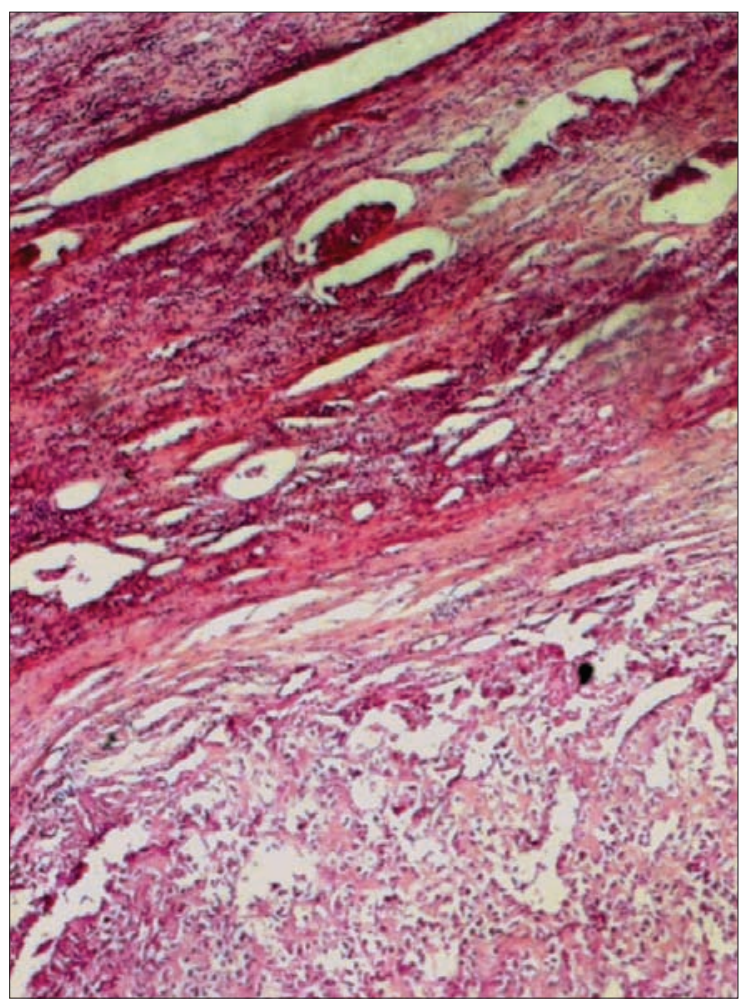

Fig. 2. Infiltration du parenchyme rénal par une prolifération sarcomateuse (coloration à l'hématoxyline-éosine $\times 100$ ). cet aspect morphologique évoquait fortement un ostéosarcome rénal. Le caractère primitif rénal a été retenu après qu'on a écarté la possibilité d'une autre origine. La patiente a fait l'objet d'un suivi régulier avec des bilans de contrôle négatifs. Dix mois plus tard, une TDM des voies urinaires pratiquée dans le cadre de la surveillance a amené à la découverte fortuite de 3 lésions métastatiques hépatiques (Fig. 5) confirmées par imagerie par résonance magnétique (IRM) au niveau du foie. Cet examen a montré des lésions nodulaires hépatiques hypervascularisées au temps artériel. Une TDM thoracique n'a pas observé de lésions secondaires aux poumons et la scintigraphie osseuse n'a révélé aucune anomalie. La patiente est actuellement en cours de traitement par chimiothérapie par doxorubicine et cisplatine avec un recul de 17 mois.

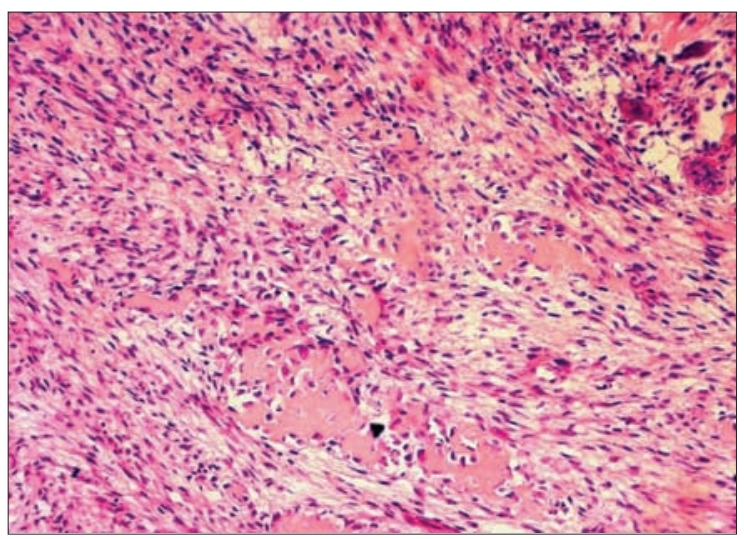

Fig. 3. Prolifération sarcomateuse produisant du tissu unostéoïde (coloration à l'hématoxyline-éosine × 200).

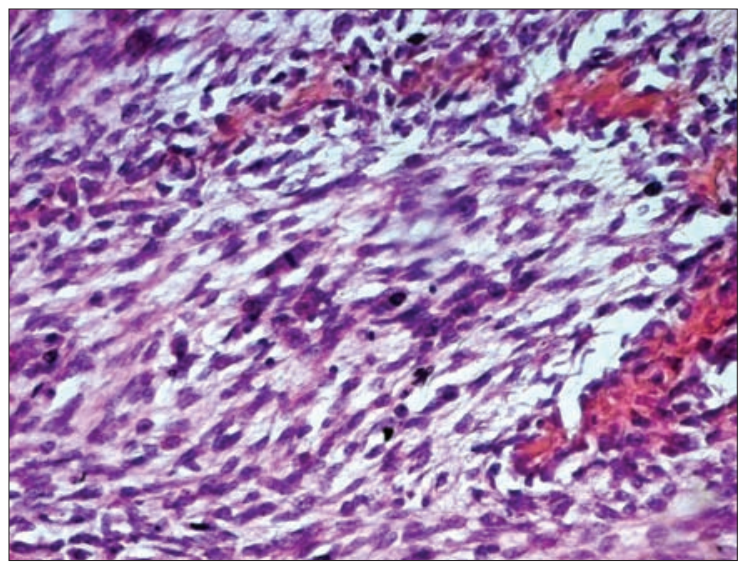

Fig. 4. Les cellules tumorales sont manifestement atypiques (coloration à l'hématoxyline-éosine $\times 400$ ). 


\section{Discussion}

Les sarcomes du rein font partie des tumeurs rares du rein. Ils représentent environ $1 \%$ de l'ensemble des tumeurs malignes du rein chez l'adulte ${ }^{3}$, le léiomyosarcome étant la forme la plus fréquente au sein de cette entité ${ }^{3,4}$.

L'ostéosarcome du rein est une pathologie extrêmement rare; une vingtaine de cas seulement ont été signalés dans la littérature mondiale. Selon les cas, l'âge de survenue est de 60 à 70 ans $^{1,5}$; notre patiente était près de la soixantaine. Le ratio par sexe est près de 1 . Les symptômes cliniques notés sont dominés par un amaigrissement, une douleur lombaire et une hématurie macroscopique, et l'examen révèle le plus souvent une masse palpable. Les symptômes cliniques sont donc semblables à ceux observés dans les autres cancers du rein. Le bilan biologique est le plus souvent normal avec parfois une élévation des phosphatases alcalines; I'analyse des urines révèle une hématurie microscopique et la cystoscopie et la cytologie urinaire sont habituellement normales ${ }^{1,2,5,6}$. Dans la majorité des cas signalés, des calcifications sont mises en évidence à l'imagerie à côté de la masse rénale, comme c'est le cas chez notre patiente. Ce type de tumeur est à haut potentiel d'extension locorégionale vers les organes voisins en raison d'un envahissement précoce de la capsule rénale, comme chez notre patiente.

Sur le plan anatomopathologique, I'Organisation mondiale de la Santé décrit cette entité comme une prolifération de cellules tumorales

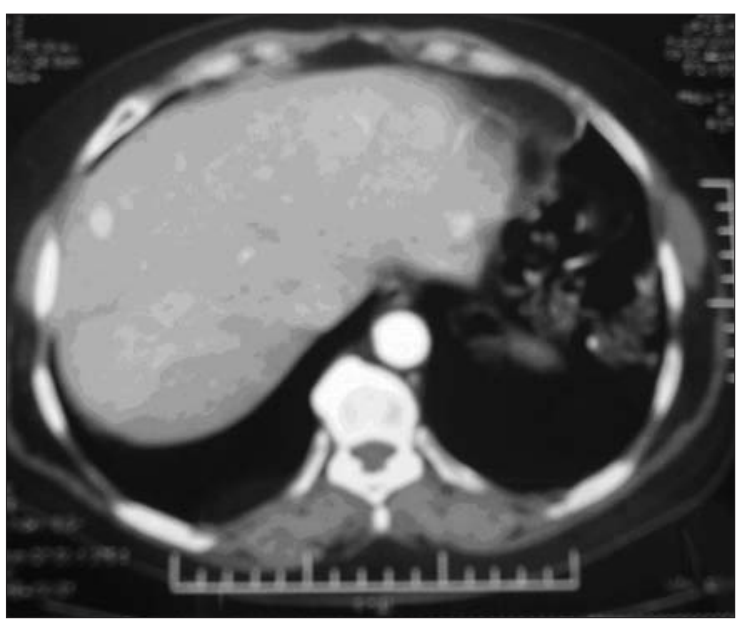

Fig. 5. Tomodensitométrie abdominale : 3 nodules hépatiques hypervascularisés des segments II, VIII, VII produisant du tissu ostéoïde au sein d'un stroma sarcomatoïde. Le diagnostic différentiel comprend essentiellement le carcinome sarcomatoïde, les formes sarcomateuses des néphroblastomes et les métastases rénales des sarcomes des tissus mous ainsi que, par extension, les sarcomes rétropéritonéaux. Il faut donc multiplier les coupes histologiques afin de trouver des structures épithéliales que l'immunohistochimie permettra de révéler ${ }^{3,7}$.

Des cas d'ostéosarcomes métastatiques au niveau du rein ont été signalés et il est important de pratiquer un bilan osseux complet pour éliminer cette possibilité diagnostique ${ }^{8,9}$. Le pronostic est très mauvais; il rejoint celui des autres sarcomes du rein. Les récidives locales et les métastases sont fréquentes et la durée médiane de la survie est de 8 à 22 mois. Les métastases peuvent se situer au niveau péritonéal, ostéomédullaire, pulmonaire et osseux. Nous signalons le premier cas, à notre connaissance, d'ostéosarcome du rein avec évolution métastatique au niveau hépatique. On a aussi signalé une ossification des métastases ${ }^{4,10-12}$.

Les différentes modalités thérapeutiques utilisées étaient la chirurgie, la radiothérapie et la chimiothérapie. Une néphrectomie élargie est nécessaire, associée à une radiothérapie du lit tumoral et à une polychimiothérapie, en cas d'envahissement des organes voisins. Il semble que la radiothérapie soit plus efficace dans les ostéosarcomes extra-squelettiques que dans les atteintes squelettiques. La chimiothérapie utilisée fait appel aux mêmes médicaments que ceux utilisés dans les ostéosarcomes des os $^{13-16}$.

Par analogie aux ostéosarcomes squelettiques, et du fait de l'apparition fréquente de métastases, le recours à une chimiothérapie néoadjuvante pourrait être bénéfique. Avant de proposer la chirurgie, il faut bien entendu confirmer le diagnostic par biopsie guidée par imagerie $d^{\prime}$ 'une masse rénale contenant des calcifications. Ce schéma thérapeutique mérite d'être validé dans des essais prospectifs.

\section{Conclusion}

Compte tenu du faible nombre de cas dans la littérature, il n'y a pas actuellement de schéma thérapeutique optimal pour la prise en charge de cette forme d'ostéosarcome. La polychimiothérapie est souvent utilisée à but curatif pour réduire le risque de récidive ou en traitement palliatif pour 
Gharbi et al.

stabiliser la maladie. Le pronostic reste toutefois très sombre à cause du diagnostic souvent tardif de la maladie. Notre cas confirme l'évolution non favorable de cette pathologie rare.

Du *Service de Médecine Carcinologique, † Service d'Anatomo-pathologie, et ¥Service de Radiologie, CHU Farhat Hached Sousse et §Service d'urologie, CHU Sahloul Sousse, Tunisie

Article évalué par les pairs.

Conflits d'intérêts : aucuns déclarés.

\section{Références}

1. Cioppa T, Marrelli D, Neri A, et al. Primary osteosarcoma of the kidney with retroperitoneal hemorhage. Case report and review of the literature. Tumori 2007;93:213-6.

2. Bigger R, Levin AS. Primary renal osteosarcoma. Urology 1995;13:674-6.

3. De Fromont $M$, Coulange C. Tumeurs rares du rein de I'adulte. Ann Urol (Paris) 2004; 38:15-23.

4. Kendal WS. The comparative survival of renal leiomyosarcoma. Can J Urol 2007;14: 3435-42.

5. Eble JN, Young RH, Störkel CS, et al. Primary osteosarcoma of the kidney: a report of three cases. J Urogen Pathol 1991;1:83-8.

6. Raby WN, Kopplin P, Weitzman S. Metastatic osteosarcoma of the kidney presenting as renal hemorrhage. J Pediatr Hematol Oncol 1996;18:321-2.
7. Messen S, Bonkhoff H, Bruch M, et al. Primary renal osteosarcoma. Case report and review of the literature. Urol Int 1995;55:158-61.

8. Ogose A, Morita T, Emura I, et al. Osteosarcoma metastatic to the kidneys without lung involvement. Jpn I Clin Oncol 1999;29:395-8.

9. O'Malley FP, Grignon DJ, Shepherd RR, et al. Primary osteosarcoma of the kidney. Report of a case studied by immunohistochemistry, electron microscopy, and DNA flow cytometry. Arch Pathol Lab Med 1991;115:1262-5.

10. Weingärtner K, Gerharz EW, Neumann K, et al. Primary osteosarcoma of the kidney. Case report and review of literature. Eur Urol 1995;28:81-4.

11. Mortensen PH. Primary osteogenic sarcoma of the kidney. Br J Urol 1989;63:101-2.

12. Tuttle RJ, Salama S, Matthews WR. Primary osteosarcoma of kidney with liposarcomatous elements. J Can Assoc Radiol 1985;36:76-8.

13. Bollack C, Cinqualbre J, Warter A, et al. Primary osteosarcoma of the kidney. Br J Urol 1982;54:435-6.

14. Axelrod R, Naidech HJ, Myers J, et al. Primary osteosarcoma of the kidney. Cancer 1978:41:724-7.

15. Fuchs N, Bielack SS, Epler D, et al. Long-term results of the cooperative German-Austrian-Swiss osteosarcoma study group's protocol COSS-86 of intensive multidrug chemotherapy and surgery of osteosarcoma of the limbs. Ann Oncol 1998;9:893-9.

16. Winkler K, Beron $G$, Delling $G$, et al. Neoadiuvant chemotherapy of osteosarcoma: results of a randomized cooperative trial (COSS-82) with salvage chemotherapy based on histological tumor response. J Clin Oncol 1988;6:329-37.

Correspondance: D` Amel Trabelsi, Service de Médecine Carcinologique CHU Farhat Hached Sousse 4000-Tunisie.; téléc. 0021673226702; trabelsiamel@yahoo.fr 